From the Laboratorio di Ricerca Area Trapianti e Oncoematologia Pediatrica, Instituto di Ricovero e Cura a Carattere Scientifico (IRCCS) Policlinico S.

Matteo; Servizio Trasfusionale, IRCCS Policlinico S. Matteo, Pavia; SC Divisione Oncologia Medica Falck, Ospedale Niguarda Ca' Granda, Milan, Italy.

Submitted May 5, 2005; accepted July 27, 2005.

Supported in part by grants from the Associazione Italiana Ricerca sul Cancro to P.C., F.L., R.M., and P.P.; Grants No. RFM/02, RFM/03, RFM/04 to P.C., F.L., R.M.; Grant No. FP6-Allostem to F.L.; and a grant from Oncologia Ca' Granda Organizzazione Non Lucrativa de Utilitá Social to P.P. and S.S. S.B. is the recipient of a grant from Associazione Morgan di

Gianvittorio.

Presented in part at the 41st Annual Meeting of the American Society of Clinical Oncology, Orlando, FL, May 13-17, 2005.

P.C. and P.P. contributed equally to this work.

Terms in blue are defined in the glossary, found at the end of this issue and online at www.jco.org.

Authors' disclosures of potential conflicts of interest are found at the end of this article.

Address reprint requests to Patrizia Comoli, MD, Laboratorio di Ricerca Area Trapianti e Oncoematologia Pediatrica, IRCCS Policlinico S. Matteo, V.le Golgi 19, 27100 Pavia, Italy; e-mail: pcomoli@ smatteo.pv.it with CC to salvatore .siena@ospedaleniguarda.it.

(c) 2005 by American Society of Clinical Oncology

0732-183X/05/2335-8942/\$20.00

DOI: $10.1200 / J C 0.2005 .02 .6195$

\title{
Cell Therapy of Stage IV Nasopharyngeal Carcinoma With Autologous Epstein-Barr Virus-Targeted Cytotoxic T Lymphocytes
}

Patrizia Comoli, Paolo Pedrazzoli, Rita Maccario, Sabrina Basso, Ornella Carminati, Massimo Labirio, Roberta Schiavo, Simona Secondino, Chiara Frasson, Cesare Perotti, Mauro Moroni, Franco Locatelli, and Salvatore Siena

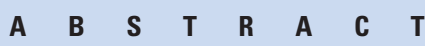

\section{Purpose}

Nasopharyngeal carcinoma (NPC) is an Epstein-Barr virus (EBV) -related malignancy expressing EBV antigens that are possible targets of cell therapy, including latent membrane protein 2 (LMP2). We conducted a clinical trial of EBV-targeted cell therapy with autologous virusspecific cytotoxic T lymphocytes (CTLs) for NPC refractory to conventional treatments.

\section{Patients and Methods}

Ten patients with EBV-related stage IV NPC in progression after conventional radiotherapy and chemotherapy received intravenously autologous EBV-specific CTLs reactivated and expanded ex vivo from peripheral blood lymphocytes through stimulation with EBV-transformed autologous B-lymphoblastoid cell lines (LCL). Toxicity, specific cellular immune responses, and clinical tumor responses were evaluated.

\section{Results}

EBV-specific CTLs could be generated in all patients and were predominantly $\mathrm{CD} 3^{+} / \mathrm{CD}^{+}$ $T$ lymphocytes displaying specific killing of autologous EBV-LCL, autologous NPC cells as well as autologous targets bearing the EBV antigen LMP2. Patients received two to 23 infusions of EBV-specific CTLs that were well tolerated with the exception of grade 1 to 2 inflammatory reactions at the tumor site in two cases. Control of disease progression was obtained in six of 10 patients (two with partial response and four with stable disease). Analysis of interferon- $\gamma-$ producing cells demonstrated an increased frequency of EBV-specific immunity, with appearance of LMP2-specific responses in four patients, of whom three had clinical benefit.

\section{Conclusion}

Cell therapy with EBV-targeted autologous CTLs is safe, induces LMP-2-specific immunologic responses, and is associated with objective responses and control of disease progression in patients with stage IV NPC resistant to conventional treatments.

\section{J Clin Oncol 23:8942-8949. (C) 2005 by American Society of Clinical Oncology}

\section{INTRODUCTION}

Nasopharyngeal carcinoma (NPC) occurs worldwide and is the third most common malignancy in southern China, where the incidence is as high as 50 per 100,000 people. ${ }^{1}$ While early stage NPC is curable with radiotherapy and chemotherapy in more than $80 \%$ of cases, the prognosis of patients with either metastatic/locally advanced at onset or recurrent disease remains poor. ${ }^{1,2}$
Second-line therapies can offer only a limited percentage of durable responses ${ }^{2}$ with relevant toxicity in patients who, in most cases, suffer from permanent sequelae of previous radiotherapy, chemotherapy, or surgery. Hence, alternative therapies capable of improving disease-free survival and associated with reduced toxicity are warranted.

Epstein-Barr virus (EBV) is present in virtually all poorly differentiated and undifferentiated nonkeratinizing NPCs regardless 
of geographic origin. ${ }^{3}$ Different from EBV-related posttransplant lymphoproliferative disease (PTLD), NPC expresses a restricted set of viral antigens, namely latent membrane protein (LMP) 1 and 2, and EBNA1, ${ }^{4}$ immunogens that are weak albeit capable of inducing a T-lymphocyte response. ${ }^{5-7}$ Because it has been shown that NPC cells are capable of immunologic processing for cytotoxic T-lymphocyte (CTL) recognition, ${ }^{8,9}$ and on the basis of the success of adoptive EBV-targeted CTL therapy for EBV-related PTLD, ${ }^{10}$ the use of anti-EBV immunotherapy strategies has been receiving increasing attention as a possible additional treatment to improve prognosis of advanced NPC. ${ }^{11-14}$ Studies of immune responses in NPC patients have demonstrated a generalized immunosuppression with decrease of cell-mediated cytolysis. ${ }^{15,16}$ In addition, it has been shown that EBV-targeted T-lymphocyte immunity is significantly reduced in NPC patients compared with healthy EBV carriers, ${ }^{12,17}$ although a measurable frequency of LMP2-specific CTL precursors (CTLp) has been detected often. ${ }^{18}$

Immunization with dendritic cells pulsed with EBVpeptides was reported to enhance specific T-lymphocyte immunity and induce partial tumor reduction in patients with advanced NPC. ${ }^{19}$ However, so far, there is limited evidence that EBV-targeted CTLs expanded from blood of NPC patients are capable of homing at the tumor site and of exerting efficient tumor killing in vivo. Our group has recently reported the case of a patient with radiotherapyand chemotherapy-resistant metastatic NPC treated with EBV-targeted CTLs reactivated from lymphocytes of an human leukocyte antigen (HLA) -matched related donor.
In this case, CTLs exhibited in vitro cytotoxicity against autologous NPC tumor cells and, once administered intravenously, induced temporary stabilization of disease, along with enhancement of patient EBV-LMP2-specific T-lymphocyte response. ${ }^{14}$ In a pilot study in China, the adoptive transfer of autologous EBV-targeted CTLs induced antiviral responses but no clinical responses in four NPC patients. ${ }^{12}$ Very recently, in a series of less advanced NPC patients, including patients treated in the adjuvant setting, autologous EBV-targeted CTL infusions proved safe and effective in promoting clinical tumor response. ${ }^{20}$

In the current study, we report the results of a trial of immunotherapy with autologous EBV-targeted CTLs in patients with stage IV radiotherapy- and chemotherapyresistant EBV-related NPC, demonstrating an enhancement of EBV-specific immune responses together with clinical responses.

\section{PATIENT AND METHODS}

\section{Patients}

Eligible patients were younger than 70 years with histologically confirmed stage IV EBV-LMP1 and/or EBV-encoded, RNA-positive NPC that was radiographically documented to be in progression despite prior conventional therapies and was not amenable to complete surgical resection or further systemic or local conventional treatments. Patients were required to have normal organ function and were excluded in case of active brain metastases, or if they had received any treatment for their disease within 30 days before enrollment, or were undergoing immunosuppressive therapy. Ten consecutive patients were enrolled on the study. Patient characteristics are reported in Table 1. Throughout the study period, the patients received EBV-specific

\begin{tabular}{|cccccccccc|}
\hline \multicolumn{7}{|c|}{ Table 1. Main Clinical Characteristics and Clinical Outcome of Patients With NPC in Progression After Conventional Therapies and Treated } \\
With EBV-Targeted CTL Therapy
\end{tabular}


CTLs as single-agent treatment. Approval for this study was obtained from the competent Institutional Review Boards at IRCCS Policlinico S. Matteo for the ex-vivo generation of EBV-targeted CTLs and at Ospedale Niguarda Ca' Granda for in-vivo treatment of NPC patients. Patients gave written informed consent before enrolment.

\section{Generation and Characterization of EBV-Specific CTLs}

Harvest and isolation of peripheral blood mononuclear cells. Because peripheral blood lymphocyte counts were low in all patients, peripheral blood mononuclear cells (PBMCs) and autologous plasma were collected through a single leukapheresis. Leukapheretic procedure was aimed at obtaining a number of PBMCs ranging from 1 to $2 \times 10^{9}$, considering, from previous experience, a minimal cellular expansion of three-fold total cellular yield reached the desired target number in all cases. PBMCs were isolated by Ficoll-Hypaque density gradient centrifugation, resuspended in X-VIVO 20 medium (BioWhittaker, Walkersville, $\mathrm{MD}$ ) with $2 \%$ autologous plasma (X-VIVO-AHS), and either used fresh or cryopreserved.

Production of autologous EBV-transformed B-lymphoblastoid cell line. PBMCs were incubated with EBV-containing supernatant from the B95-8 cell line (American Type Culture Collection, Rockville, MD) in the presence of $800 \mathrm{ng} / \mathrm{mL}$ of cyslosporine-A (Sandoz Pharmaceuticals, Basel, Switzerland). Cells were continuously cultured for 3 to 4 weeks, following a protocol previously described. ${ }^{21}$

Preparation of autologous EBV-specific CTL lines. EBVspecific CTLs were reactivated and expanded in vitro according to a method previously reported, following good laboratory practice (GLP) standard procedures. ${ }^{22}$ Before cryopreservation, $\mathrm{T}$ cells were examined for immunophenotype, sterility, and EBV specificity in a standard ${ }^{51} \mathrm{Cr}$-release assay against a panel of targets including autologous B-lymphoblastoid cell line (LCL), autologous phytohemagglutinin (PHA) blasts pulsed with $2 \mu \mathrm{g} / \mathrm{mL}$ of a peptide mix containing 15-mer peptides spanning the EBV LMP2 protein (Jerini, Berlin, Germany), and autologous tumor cells. Tumor cells were obtained from a baseline tumor biopsy, according to a previously published method. ${ }^{14}$ Early-passage neoplastic cells were used to minimize the possibility of loosing primary characteristics as a consequence of extensive in vitro reculturing.

Flow cytometry. Monoclonal antibodies used to characterize cultured cells were CD3 (anti-Leu-4) flourescein isothiocyanate (FITC), anti-HLA-DR phycoerythrin (PE), CD8 (anti-Leu-2a) FITC and PE, CD56 (anti-Leu-19) PE, anti-TRC $\gamma \delta$ FITC, CD4 (anti-Leu-3a) PE, CD19 (anti-Leu-12) FITC, CD20 (anti-Leu16) PE, CD45 (anti-HLe-1) FITC (Becton Becton Dickinson, Mountain View, CA). Appropriate isotype-matched controls were included. Cytofluorimetric analysis was performed by means of direct immunofluorescence on a FACScan flow cytometer (Becton Becton Dickinson).

\section{EBV-Specific CTL Infusion Schedule and Patient Evaluation}

Cryopreserved CTLs were thawed rapidly and administered intravenously. Patients were monitored for vital signs before and immediately after each infusion. Autologous CTLs were infused at weekly intervals for the first four administrations and subsequently every 2 to 4 weeks. For the first five patients, CTL infu- sion schedule included a first dose of $2 \times 10^{7} \mathrm{CTL} / \mathrm{m}^{2}$ of body surface, followed by subsequent doses of $4 \times 10^{7} \mathrm{CTL} / \mathrm{m}^{2}$. This infusion scheme was derived from that employed in the treatment of EBV-associated lymphoproliferative disease after transplantation. ${ }^{10,21}$ In the last five patients (patients 6 to 10), the schedule was modified to deliver a higher number of CTLs, and the patients received four escalating doses of EBV CTL $\left(2 \times 10^{7}, 4 \times 10^{7}, 6 \times 10^{7}\right.$, and $\left.8 \times 10^{7} \mathrm{CTL} / \mathrm{m}^{2}\right)$ every 2 weeks, followed by infusions of $6 \times 10^{7} \mathrm{CTL} / \mathrm{m}^{2}$ every 2 to 4 weeks, accompanied by administration of low-dose recombinant interleukin-2 (IL-2; Chiron, Milan Italy; $1 \times 10^{6} \mathrm{U}$ subcutaneously qd) with the aim of prolonging T-lymphocyte life span. We planned to deliver the first five to six doses of CTLs until disease evaluation, and continue with CTL infusions in case of evidence of clinical benefit. In all patients, cell therapy was discontinued upon evidence of disease progression.

Disease evaluation was performed according to the criteria proposed by the Response Evaluation Criteria In Solid Tumors (RECIST) Committee 22 after 1 month and every 2 months thereafter. To evaluate the effects of CTL administration on the frequency of IFN- $\gamma$ secreting lymphocytes and on EBV-directed cytotoxic activity, peripheral blood samples were collected at baseline, and at different times after CTL infusions. EBV DNA levels were monitored by $\mathrm{PCR}^{21}$ on plasma samples.

\section{Enzyme-Linked Immunospot Assay}

For enzyme-linked immunospot (ELISPOT) assay, 96-well multiscreen filter plates (MAIPS 4510, Millipore, Bedford, MA) were coated with $100 \mu \mathrm{L}$ of primary antibody (IFN- $\gamma$, Mabtech, Nacka, Sweden) at $2.5 \mu \mathrm{g} / \mathrm{mL}$, and incubated overnight at $4^{\circ} \mathrm{C}$. Peripheral blood mononuclear cells (PBMCs) were thawed and cultured overnight in RPMI medium with $10 \%$ fetal calf serum before use in the assay, and were then seeded in the presence of EBV-LCL or $2 \mu \mathrm{g} / \mathrm{mL}$ of the EBV LMP-2 peptide mix. After incubation for 24 hours at $37^{\circ} \mathrm{C}, 100 \mu \mathrm{L}$ of biotinylated secondary antibody (Mabtech, $0.5 \mu \mathrm{g} / \mathrm{mL}$ ) was added, and plates were then processed according to standard procedure. ${ }^{23}$ IFN- $\gamma$-producing spots were counted using a Elispot reader (Bioline, Torino, Italy). The number of spots per well was calculated after subtraction of assay background, quantitated as average of 24 wells containing only sterile complete medium, and specific backgrounds, quantitated as the sum of cytokine spots associated with responders alone, EBV-LCL alone, responders plated with dimethyl sulfoxide solvent control, as appropriate.

\section{LMP2-Specific Cytotoxicity Assay}

Specific cytotoxic activity before and after CTL infusion was assessed according to a method previously described. ${ }^{13}$ In brief, cryopreserved PBMCs were thawed and incubated for 24 hours with $10 \mathrm{U} / \mathrm{mL}$ rIL-2 and $2 \mu \mathrm{g} / \mathrm{mL}$ of the EBV LMP2 peptide mix, after depletion of $\mathrm{CD}_{16}{ }^{+}$and $\mathrm{CD}_{5} 6^{+}$cell populations. As targets, 3 -day PHA blasts were pulsed with $2 \mu \mathrm{g} / \mathrm{mL}$ of the EBV LMP2 peptide mix or with control peptide.

\section{RESULTS}

\section{Biologic Characterization of EBV-Targeted Autologous CTL Lines Utilized for Cell Therapy}

EBV-transformed autologous B-LCL and autologous EBV-specific CTL lines were successfully generated ex 
vivo from all 10 patients. Growth kinetics of the T-cell lines from our cohort of NPC patients with disease progression corresponded to a median five-fold cell expansion at 4 weeks (range, 2 to 17 weeks), thus comparable to what was observed previously for healthy donors. ${ }^{24}$

Phenotypic flow cytometry analysis indicated that the CTL lines generated from PBMCs of NPC patients were heterogeneous with respect to the percentages of $\mathrm{CD}^{+} /$ $\mathrm{CD}^{+}{ }^{+}, \mathrm{CD}^{+} / \mathrm{CD}^{+}$and $\mathrm{CD} 4 / \mathrm{CD} 8$ ratio (Table 2 ). In detail, $\mathrm{CD}^{+}$cells ranged from $30 \%$ to $89 \%$ (median, $67 \%$ ), $\mathrm{CD}^{+}{ }^{+}$lymphocytes ranged from $8 \%$ to $68 \%$ (median $29 \%$ ), and $\mathrm{CD}^{+} / \mathrm{HLA}-\mathrm{DR}+$ population was between $54 \%$ and $96 \%$ (median $87 \%$ ). Moreover, the CTL lines contained a median of $20 \%$ cells that were $\mathrm{CD}^{+} /$ $\mathrm{CD}^{+} / \mathrm{CD} 6^{+}$and $5 \%$ cells with natural killer phenotype $\left(\mathrm{CD}_{5} 6^{+} / \mathrm{CD}^{-}\right)$. The proportion of TCR $\gamma \delta+$ cells was very low in all patients.

To test EBV specificity of CTLs, we measured cytotoxicity against autologous LCLs or HLA-mismatched LCLs, as well as against allogeneic PHA blasts. CTLs from NPC patients contained HLA-restricted components, since lysis of autologous LCLs was always higher than that of HLA-mismatched LCLs (Table 2). No lysis against allogeneic PHA blasts was observed. Activity of EBV CTL lines against LMP2 protein was present in five of the 10 patients (Fig 1).

The presence of in vitro activity of CTLs against autologous NPC cells, possibly predictive of an in vivo antitumor effect, was also assessed whenever autologous tumor cell lines were available. The level of cytotoxicity against patient tumor cells, tested in four patients, ranged between $7 \%$ and 20\% (median, 15\%) at an effector-totarget ratio of 10:1 (Fig 1).

\section{Clinical Effects of Autologous EBV-Targeted CTL Therapy}

Patients enrolled in this trial received a median of 10 CTL administrations (range, 2 to 23). Hospital admission was not required. During CTL administration, no acute adverse effects occurred. One to 2 days after each CTL administration, patient 2 had grade 2 inflammation at the tumor site (suvraclavear and laterocervical nodes) requiring antiinflammatory medication, including steroids, and causing treatment delays. Patient 10 had an inflammatory reaction at the site of neck lymph node involvement at the time of the 12th CTL administration. No other late adverse effect ascribable to CTL therapy was observed in the remaining patients. Two patients receiving IL-2 experienced mild adverse effects related to this drug (ie, malaise, fever, chills), that in one case led to IL-2 discontinuation because of poor compliance. In all patients, cell therapy was discontinued upon evidence of disease progression.

Clinical response to CTL therapy is summarized in Table 1. Four patients achieved stable disease (SD) lasting a median of 6 months (range, 4 to 15 months). Patient 10 persists in SD at the time of this writing. Patients 2 and 7 achieved partial response (PR) 3 and 4 months after the first CTL infusion, respectively. Patient 2, notwithstanding the achievement of a PR, refused to continue CTL therapy, and was lost to follow-up. Patient 7 presented with massive liver metastases involving more than $75 \%$ of the organ. Within 24 hours from the first CTL infusion, an increase in the levels of plasma EBV DNA levels was observed, from a baseline value of 1,310 copies/ $\mu \mathrm{L}$ to 2,136 copies/ $\mu \mathrm{L}$, likely due to lysis of tumor cells bearing the viral genome. Three weeks after the fourth infusion, EBV DNA levels were 940 copies/ $\mu$ L. During the first month of CTL therapy a reduction of the levels of EBV DNA, liver necrosis and cholestasis enzymes was observed, together with an improvement of the performance status from 2 to 0 . Tumor regression was confirmed by computed tomography (CT) scan and CT-aided positron emission tomography (PET) imaging (Fig 2). Eventually, tumor progression occurred after 5 months and the patient died as a result of liver failure.

\begin{tabular}{|c|c|c|c|c|c|c|c|c|c|c|}
\hline $\begin{array}{l}\text { Patient } \\
\text { No. }\end{array}$ & UPN & $\begin{array}{c}\mathrm{CD}^{+} / \mathrm{CD}^{+}{ }^{+} \\
\text {Cells }(\%)\end{array}$ & $\begin{array}{c}\mathrm{CD}^{+} / \mathrm{CD}^{+} \\
\text {Cells }(\%)\end{array}$ & $\begin{array}{c}\mathrm{CD}^{+} / \mathrm{DR}^{+} \\
\text {Cells }(\%)\end{array}$ & $\begin{array}{l}\text { TCR } \gamma \delta+ \\
\text { Cells (\%) }\end{array}$ & $\begin{array}{l}\mathrm{CD}^{+} / 56^{+} \\
\text {Cells }(\%)\end{array}$ & $\begin{array}{c}\mathrm{CD}^{\mathrm{CD}^{+}} / \mathrm{CD}^{-} \\
\text {Cells }(\%)\end{array}$ & $\begin{array}{l}\text { Auto LCL } \\
\text { (\% lysis) }\end{array}$ & $\begin{array}{l}\text { Allo } L C L \\
\text { (\% lysis) }\end{array}$ & $\begin{array}{l}\text { Allo PHA } \\
\text { (\% lysis) }\end{array}$ \\
\hline 1 & 10002348 & 28 & 70 & 87 & 4 & 8 & 2 & 73 & 13 & 3 \\
\hline 2 & 30208742 & 41 & 65 & 87 & 7 & 16 & 0 & 33 & 4 & 1 \\
\hline 3 & 30233207 & 47 & 30 & 54 & 3 & 10 & 30 & 49 & 5 & 5 \\
\hline 4 & 30345153 & 34 & 52 & 70 & 5 & 21 & 26 & 38 & 20 & 0 \\
\hline 5 & 10043598 & 29 & 70 & 91 & 5 & 20 & 10 & 37 & 10 & 0 \\
\hline 6 & 30110679 & 9 & 63 & 84 & 1 & 20 & 32 & 39 & 28 & 1 \\
\hline 7 & 30486646 & 68 & 32 & 88 & 2 & 6 & 7 & 61 & 19 & 2 \\
\hline 8 & 30287851 & 8 & 89 & 92 & 4 & 30 & 3 & 64 & 17 & 2 \\
\hline 9 & 30376608 & 10 & 87 & 95 & 2 & 20 & 3 & 32 & 9 & 1 \\
\hline 10 & 30545247 & 30 & 69 & 96 & 10 & 40 & 2 & 96 & 10 & 0 \\
\hline Median & & 29 & 67 & 87 & 4 & 20 & 5 & 44 & 11 & 1 \\
\hline Range & & $8-68$ & $30-89$ & $54-96$ & $1-10$ & $6-40$ & $0-32$ & $32-96$ & $4-28$ & $0-5$ \\
\hline
\end{tabular}

NOTE. The cytotoxicity results are reported for an effector to target ratio of 10:1.

Abbreviations: EBV, Epstein-Barr virus; CTL, cytotoxic T lymphocyte; NPC, nasopharyngeal carcinoma; UPN, unique patient number; LCL, B-lymphoblastoid cell line; PHA, phytohemagglutinin. 


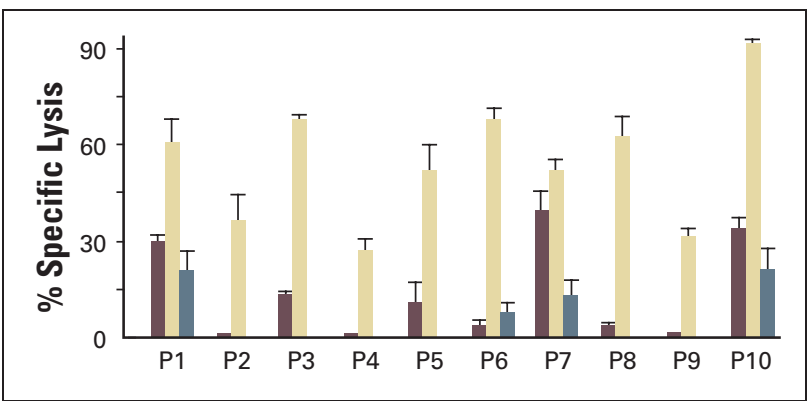

Fig 1. Functional characteristics of the infused Epstein-Barr virus-targeted cytotoxic T-lymphocyte (CTL) lines. Cytotoxicity of CTL reactivated from the 10 patients, in response to autologous EBV-B-lymphoblastoid cell lines (gold bars), autologous EBV LMP-2 protein peptide mix-pulsed PHA blasts (brown bars), and autologous tumor cells (blue bars) is reported. Results represent mean cytotoxicity at a 10:1 effector to target (E:T) ratio \pm standard deviation.

\section{Immunologic Effects of EBV-Targeted Autologous CTL Therapy}

To determine the effect of CTL infusions on specific immunity, frequency of IFN- $\gamma$-producing cells and specific cytotoxic capacity were evaluated on samples obtained at different times after T-cell transfer. All patients had detectable, albeit very low in comparison to healthy EBV-seropositive donors, ${ }^{24}$ levels of EBV-LCL-specific
IFN- $\gamma$-producing cells before CTL therapy (Fig 3B). Upon T-cell transfer, the frequency of LCL-specific T cells increased (Fig 3B).

Before EBV-specific CTL therapy, levels of LMP2specific IFN- $\gamma$-secreting cells were undetectable or very low in all patients $\left(<2 / 10^{5}\right.$ PBMCs; Fig $\left.3 \mathrm{~A}\right)$. After two to four infusions (ie, 2 to 4 weeks after the first CTL dose), four of the 10 patients (patients 1, 3, 7, and 10) showed a measurable response, that initially increased with the number of CTL infusions, the mean peak frequency observed being $34 / 10^{5}$ PBMCs (Fig 3A). Patient 2, who had a clinical response to treatment, and patients 4 and 9, who showed tumor stabilization, did not reveal detectable levels of LMP2-specific T cells in their peripheral blood. We then proceeded to evaluate whether the LMP2-specific IFN- $\gamma$-producing cells present in the PBMC of the patients after CTL therapy were also endowed with cytotoxic capacity. In line with the IFN- $\gamma$ production data, PBMC collected postinfusion from patients 3 and 7 displayed measurable lytic activity against LMP2-bearing targets compared with cells harvested at baseline (cytotoxicity at a 10:1 effector-to-target ratio: $8 \pm 1$ and $10 \pm 2$, respectively), whereas lymphocytes
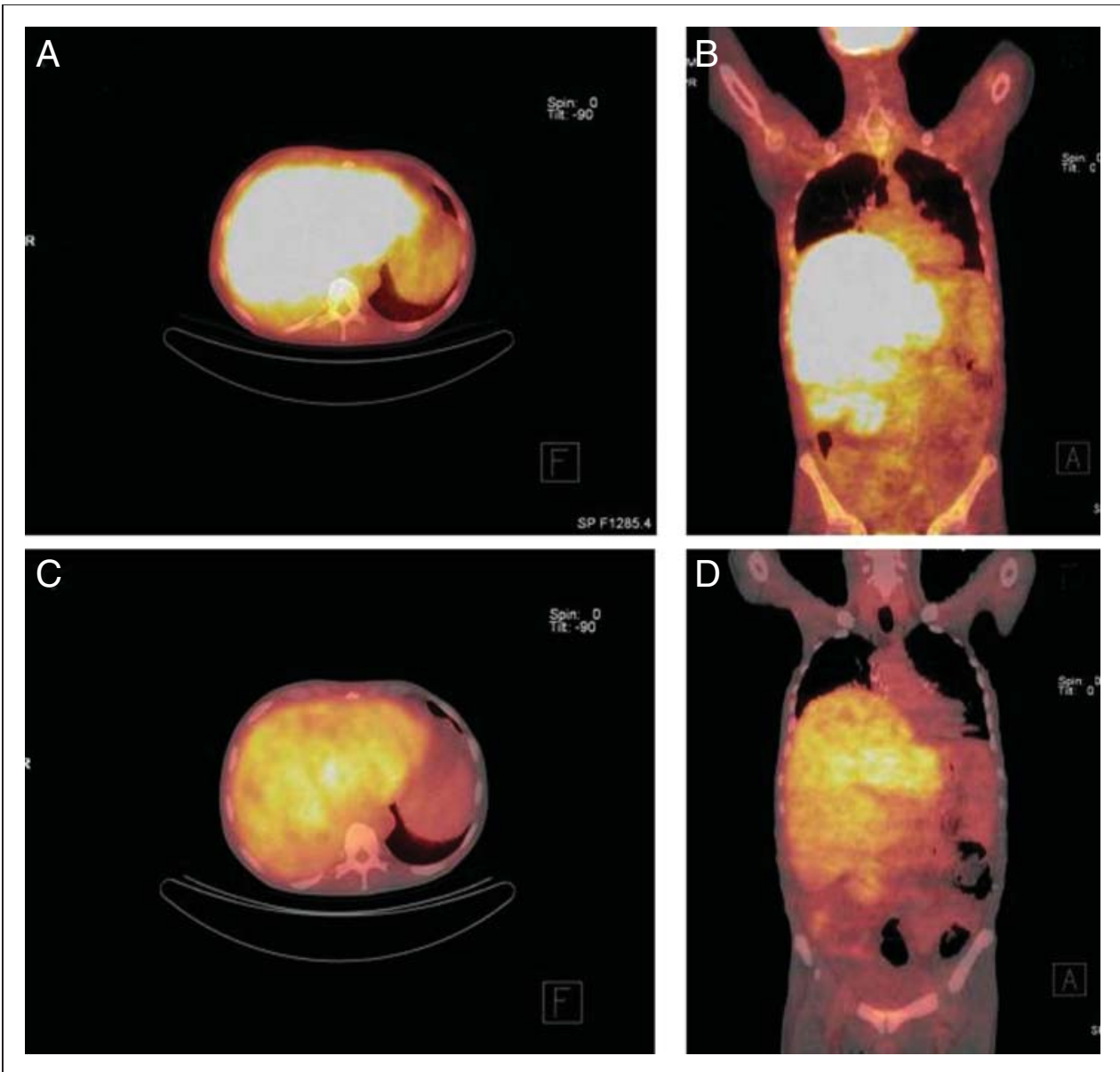

Fig 2. Objective response to EpsteinBarr virus (EBV) -targeted cytotoxic T-lymphocyte (CTL) therapy in patient 7. (A and B) Computed tomographyaided positron emission tomography imaging before T-cell therapy shows massive liver involvement. ( $C$ and $D$ ) After 3 months of EBV-targeted CTL therapy, reduction of size and isotope uptake of NPC lesions was detected. 


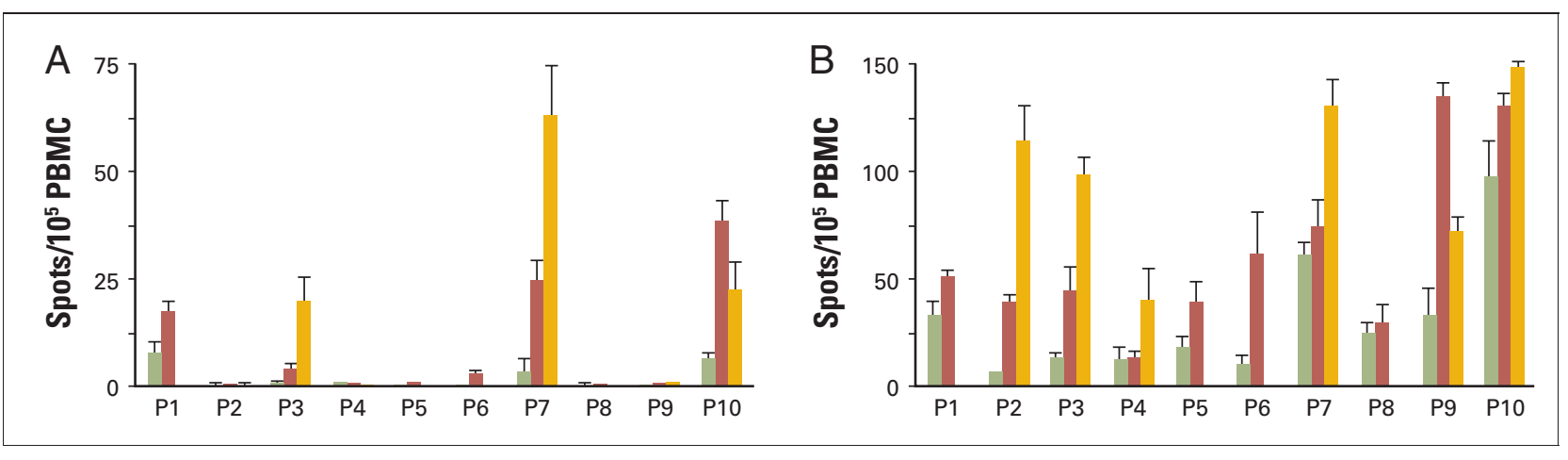

Fig 3. Immunologic effect of Epstein-Barr virus (EBV)-targeted cytotoxic T-lymphocyte (CTL) therapy on patient EBV-specific interferon- $\gamma$ (IFN- $\gamma$ ) production. IFN$\gamma$-secreting lymphocytes were measured in peripheral blood mononuclear cells (PBMCs) obtained from the 10 patients before (green bars), 4 weeks (red bars), and 8 weeks (orange bars) after CTL therapy, in response to (A) EBV-latent membrane protein 2 protein peptide mix and (B) EBV-B-lymphoblastoid cell lines (LCL).

obtained from patients 2 and 5 did not show lysis of LMP2-bearing targets.

In all patients, the levels of both EBV-LCL-specific and LMP2-specific cells declined over time (data not shown).

\section{DISCUSSION}

In the present study, we demonstrate that clinical and immunologic responses can be obtained in patients with radiotherapy- and chemotherapy-resistant stage IV EBVrelated NPC by administration of EBV-targeted autologous polyclonal CTL therapy. In particular, we document that despite multiple lines of previous immunosuppressive treatments, it is feasible to reactivate and expand ex vivo CTLs that possess normal cytotoxic activity against autologous EBV-infected B-cell lines. Moreover, EBV-targeted CTL administered intravenously appear safe also in heavily pretreated patients with bulky disease. More importantly, even though the patients enrolled had progressive, stage IV NPC after failure of conventional treatments, tumor control related to cell therapy did occur.

Clinical responses consisting of objective partial remission occurred in two patients, whereas four patients maintained stable disease for an overall $60 \%$ control of disease progression. This figure represents a remarkable clinical result if one considers that patients enrolled in this study were indeed eligible for supportive care only, and had an extensive tumor burden. In addition, CTL therapy was well tolerated, with mild adverse effects in a minority of patients.

For the two patients showing a reduction of tumor burden, it was not possible to obtain a postinfusion biopsy. Therefore, we could not perform a T-cell receptor repertoire analysis by CDR3 spectratyping and sequencing, which would have provided information on a possible homing of infused CTL at the tumor site through characterization of T-cell clonotypes present in the lesion after T-cell infusion, and a comparison to clonotypes included in the transferred CTL line. However, in these patients showing objective responses, autologous CTL therapy was associated with either symptoms suggestive of CTL trafficking at the tumor site (patient 2) or an increase of EBV-DNA plasma levels, reminiscent of tumor lysis (patient 7). These latter observations provide further evidence suggesting that infused CTLs were directly responsible for tumor reduction.

Immunologic responses to CTL therapy occurred in all patients, with four of 10 patients showing a boost of LMP2-specific cell frequency detected in peripheral blood. These LMP2-specific cells were functional, since they produced IFN- $\gamma$ and displayed some lytic activity against LMP2-bearing autologous NPC targets. It is noteworthy that, in three of the four patients, the development of an LMP2-specific response coincided with a clinical benefit. For the remaining patients, in whom a clinical response was observed in the absence of a detectable enhancement of LMP2-specific immunity in the periphery, we hypothesize that either CTLs, homing at the tumor site, were not recirculating in the peripheral blood, or that the immune response was directed towards LMP1 or EBNA 1 antigens, ${ }^{20}$ which we could not test, rather than LMP2.

We delivered CTLs weekly for the first month, and subsequently at 2- to 4-week intervals in the attempt to augment efficacy of T-cell therapy based on the knowledge that autologous $\mathrm{CD}^{+} \mathrm{T}$ cell clones infused in patients with melanoma persisted for a median of 7 days after transfer. ${ }^{25}$ Moreover, since the administration of systemic IL-2 appears to prolong in vivo persistence of infused lymphocytes, ${ }^{25}$ we further implemented our treatment protocol by using low-dose IL-2 in five patients. Notwithstanding the increased anti-LMP2 immunity occurring in some patients, and the attempts to prolong CTL survival in vivo, the clinical response in our patients was often transient. This may be related to the large tumor burden and advanced stage of disease of the patients enrolled in this study, which might have favored treatment-induced selection for antigen loss variants, associated to a generally low activity of CTL lines against autologous tumor cells. 
In our study, we employed CTLs stimulated with EBV-LCL. The lines thus obtained potentially include specificities towards any latent antigen, albeit with a preferential selection of cells directed against immunodominant proteins, such as EBNA3. Since NPC tumor cells express a restricted pattern of EBV antigens, antitumor responses could be further enhanced by strategies aimed at increasing the number of LMP2 and/or LMP1-specific T cells in the infusion product. In particular, enrichment of subdominant antigen specificities through either stimulation with dendritic cells expressing LMP2 and/or LMP1 and/or EBNA1, ${ }^{26,27}$ or expansion of LCL-stimulated CTL after vaccination of patients with LMP2 peptide-pulsed dendritic cells ${ }^{13}$ or LMP1 polyepitope adenovirus vaccine, ${ }^{28}$ could be obtained. The number of cells in each dose that we administered in this phase I trial was relatively small, if compared to the magnitude of infusions reported in other studies of T-cell therapy for cancer. ${ }^{29}$ Thus, an additional improvement to our current protocol will be the administration of higher CTL doses. In addition, the potential for CTL expansion in vivo may be augmented in the condition of profound lymphopenia, such as following the administration of lymphoablative chemotherapy. ${ }^{29}$

Overall, our phase I-II study document that the intravenous administration of autologous EBV-specific CTLs is safe, augments EBV-specific immune responses, and pro- duces clinical responses in patients with stage IV NPC. However, as also demonstrated for the treatment of EBV-related PTLD, ${ }^{30}$ CTLs used as monotherapy may have the same limitations described for chemotherapy, namely selection of malignant clones with poor expression of EBV antigens, and thus refractory to CTL killing. Based on original evidences presented and discussed in the preceding sections, we intend to pursue further studies of cell therapy with EBV-targeted CTLs either earlier in the course of NPC disease or in association with other conventional therapeutic modalities, ${ }^{30,31}$ with the aim of reducing the risk of tumor selection and thus ameliorating the clinical outcome.

\section{Acknowledgment}

We thank Angelo Vanzulli for radiology consultations; Claudio Rossetti for nuclear medicine consultations; Andrea Ferrari, Maura Massimino, and Giovanna Gelosa for patient referral; and Pier Luigi Oreste and Marco Paulli for histopathologic studies.

\section{Authors' Disclosures of Potential Conflicts of Interest}

The authors indicated no potential conflicts of interest.

\section{REFERENCES}

1. Chan AT, Teo PM, Johnson PJ: Nasopharyngeal carcinoma. Ann Oncol 13:1007-1015, 2002

2. Forastiere $A$, Koch W, Trotti $A$, et al: Head and neck cancer. N Engl J Med 345:1890-1900, 2001

3. Thompson MP, Kurzrock R: Epstein Barr virus and cancer. Clin Cancer Res 10:803-821, 2004

4. Cohen JI: Epstein-Barr virus infection. N Engl J Med 343:481-492, 2000

5. Khanna R, Burrows SR, SteigerwaldMullen PM, et al: Targeting Epstein-Barr virus nuclear antigen 1 (EBNA1) through the class II pathway restores immune recognition by EBNA1-specific cytotoxic T lymphocytes: Evidence for HLA-DM-independent processing. Int Immunol 9:1537-1543, 1997

6. Meij $P$, Leen $A$, Rickinson $A B$, et al: Identification and prevalence of $\mathrm{CD} 8+\mathrm{T}$-cell responses directed against Epstein-Barr virusencoded latent membrane protein 1 and latent membrane protein 2. Int J Cancer 99:93-99, 2002

7. Rickinson AB, Moss DJ: Human cytotoxic $T$ lymphocyte responses to Epstein-Barr virus infection. Annu Rev Immunol 15:405-431, 1997

8. Khanna R, Busson $P$, Burrows $S$, et al: Molecular characterisation of antigen-processing function in nasopharyngeal carcinoma (NPC): Evidence for efficient presentation of Epstein-
Barr virus cytotoxic T cell epitopes by NPC cells. Cancer Res 58:310-314, 1998

9. Lee SP, Chan ATC, Cheung S, et al: CTL control of EBV in nasopharyngeal carcinoma (NPC): EBV-specific CTL responses in the blood and tumors of NPC patients and the antigenprocessing function of the tumor cells. J Immunol 165:573-582, 2000

10. Straathof KC, Savoldo B, Heslop HE, et al: Immunotherapy for post-transplant lymphoproliferative disease. $\mathrm{Br} \mathrm{J}$ Haematol 118:728-740, 2002

11. Khanna R, Moss DJ, Burrows SR: Vaccine strategies against Epstein-Barr virus-associated diseases: Lessons from studies on cytotoxic T-cell-mediated immune regulation. Immunol Rev 170:49-64, 1999

12. Chua D, Huang J, Zheng B, et al: Adoptive transfer of autologous Epstein-Barr virus- specific cytotoxic $T$ cells for nasopharygeal carcinoma. Int J Cancer 94:73-80, 2001

13. Lin $C$, Lo W, Lee $T$, et al: Immunization with Epstein-Barr virus (EBV) peptide-pulsed dendritic cells induces functional CD8+ T-cell immunity and may lead to tumor regression in patients with EBV-positive nasopharyngeal carcinoma. Cancer Res 62:6952-6958, 2002

14. Comoli $P$, De Palma $R$, Siena $S$, et al: Adoptive transfer of allogeneic EBV-specific cytotoxic $T$ cells with in vitro antitumor activity boosts LMP-2-specific immune response in a patient with EBV-related nasopharyngeal carcinoma. Ann Oncol 15:113-117, 2004

15. Laytragoon-Lewin N, Porwit-MacDonald A, Mellstedt $\mathrm{H}$, et al: Alteration of cellular mediated cytotoxicity, T cell receptor zeta (TCRz) and apoptosis related gene expression in nasopharyngeal carcinoma (NPC) patients: Possible clinical relevance. Anticancer Res 20:10931100, 2000

16. Chan SH, Chew TS, Goh EH, et al: Impaired general cell-mediated immune functions in vivo and in vitro in patients with nasopharyngeal carcinoma. Int $\mathrm{J}$ Cancer 18: 139-144, 1976

17. Moss DJ, Chan SH, Burrows SR, et al: Epstein-Barr virus $T$ cell response in nasopharyngeal carcinoma patients. Int J Cancer 32:301305, 1983

18. Whitney BM, Chan ATC, Rickinson AB, et al: Frequency of Epstein-Barr virus-specific cytotoxic T lymphocytes in the blood of Southern Chinese blood donors and nasopharyngeal carcinoma patients. J Med Virol 67:359-363, 2002

19. Lin C, Lo W, Lee $T$, et al: Immunization with Epstein-Barr virus (EBV) peptide-pulsed dendritic cells induces functional CD8+ T-cell immunity and may lead to tumor regression in patients with EBV-positive nasopharyngeal carcinoma. Cancer Res 62:6952-6958, 2002

20. Straathof KC, Bollard CM, Popat U, et al: Treatment of nasopharyngeal carcinoma with Epstein-Barr virus-specific T lymphocytes. Blood 105:1898-1904, 2005

21. Comoli $P$, Labirio $M$, Basso $S$, et al: Infusion of autologous Epstein-Barr virus (EBV)specific cytotoxic T cells for prevention of EBVrelated lymphoproliferative disorder in solid organ transplant recipients with evidence of 
active virus replication. Blood 99:2592-2598, 2002

22. James K, Eisenhauer E, Christian M, et al: Measuring response in solid tumors: Unidimensional versus bidimensional measurement. J Natl Cancer Inst 91:523-528, 1999

23. Yang J, Lemas VM, Flinn IW, et al: Application of the ELISPOT assay to the characterization of $\mathrm{CD} 8+$ responses to Epstein-Barr virus antigens. Blood 95:241-248, 2000

24. Savoldo B, Huls MH, Liu Z, et al: Autologous Epstein-Barr virus (EBV)-specific cytotoxic $T$ cells for the treatment of persistent active EBV infection. Blood 100:4059-4066, 2002

25. Yee C, Thompson JA, Byrd D, et al: Adoptive $T$ cell therapy using antigen-specific $\mathrm{CD} 8+\mathrm{T}$ cell clones for the treatment of patients with metastatic melanoma: In vivo persistence, migration, and antitumor effect of transferred $T$ cells. Proc Natl Acad Sci U S A 99:1616816173, 2002

26. Gottschalk S, Edwards OL, Sili U, et al: Generating CTLs against the subdominant Epstein-Barr virus LMP1 antigen for the adoptive immunotherapy of EBV-associated malignancies. Blood 101:1905-1912, 2003

27. Taylor GS, Haigh TA, Gudgeon $\mathrm{NH}$ et al: Dual stimulation of Epstein-Barr virus (EBV)-specific CD4+- and CD8+-T-cell responses by a chimeric antigen construct: Potential therapeutic vaccine for EBV-positive nasopharyngeal carcinoma. J Virol 78:768-778, 2004

28. Duraiswamy J, Bharadwaj $M$, Tellam J, et al: Induction of therapeutic T-cell responses to subdominant tumor-associated viral oncogene after immunization with replication-incompetent polyepitope adenovirus vaccine. Cancer Res 64:1483-1489, 2004

29. Dudley ME, Wunderlich JR, Yang JC, et al: Adoptive cell transfer therapy following nonmyeloablative but lymphodepleting chemotherapy for the treatment of patients with refractory metastatic melanoma. J Clin Oncol 23:23462357, 2005

30. Comoli $P$, Maccario $R$, Locatelli $F$, et al: Treatment of EBV-related post-renal transplant lymphoproliferative disease with a tailored regimen including EBV-specific T cells. Am J Transplant 5:1418-1422, 2005

31. Lake RA, Robinson BWS: Immunotherapy and chemotherapy: A practical partnership. Nat Rev Cancer 5:397-405, 2005 